\title{
Utilisation de suppléments alimentaires et apport en fer, en zinc et en folate chez les femmes enceintes de London, Ontario
}

\author{
A. Roy, M. Sc. (1, 2); S. E. Evers, Ph. D. (3); M. K. Campbell, Ph. D. (1, 4, 5, 6, 7)
}

Cet article a fait l'objet d'une évaluation par les pairs.

\section{Résumé}

Introduction : Nous avons examiné l'apport alimentaire en fer, en zinc et en folate, estimé à partir d'aliments et de suppléments, chez 2019 femmes enceintes ayant participé au Prenatal Health Project (PHP). Le PHP a recruté des femmes enceintes dans des cliniques d'échographie de London (Ontario) entre 2002 et 2005.

Méthodologie : Les participantes ont répondu à une enquête téléphonique qui comprenait un questionnaire sur la fréquence de consommation des aliments, ainsi que des questions sur l'utilisation de suppléments alimentaires, ce qui nous a permis d'établir les fréquences d'utilisation de suppléments alimentaires. Nous avons estimé les valeurs d'apport nutritionnel à partir des aliments et des suppléments puis nous les avons additionnées pour calculer l'apport quotidien total.

Résultats : La plupart des femmes prenaient un supplément de multivitamines, et elles étaient nombreuses à prendre des suppléments d'acide folique et de fer; toutefois, un cinquième de l'échantillon n'utilisait aucun supplément contenant l'un de ces trois micronutriments. Malgré le fait que, dans l'ensemble, ces femmes bénéficiaient d'un statut socio-économique élevé, une proportion importante de la cohorte affichait des valeurs inférieures aux valeurs de l'apport nutritionnel recommandé pour le fer, le zinc et le folate. Ces résultats laissent croire que d'autres obstacles auraient une incidence sur les habitudes alimentaires.

Conclusions : Il faut pousser plus loin la recherche quant à la façon de mieux promouvoir l'utilisation de suppléments et une saine alimentation durant la grossesse.

Mots-clés : fer, folate, zinc, supplément alimentaire, alimentation, nutrition, grossesse

\section{Introduction}

La prise de nutriments en quantité suffisante durant la grossesse est essentielle pour la santé de la mère, du fœtus et de l'enfant. Toutefois, peu d'études en population ont examiné l'apport alimentaire et l'utilisation de suppléments alimentaires chez les femmes enceintes au Canada. Le fer, le zinc et le folate retiennent particulièrement l'attention. Le fer est indispensable à la structure et à la fonction des globules rouges; une carence en fer peut entraîner l'anémie. L'anémie et la carence en fer durant la grossesse peuvent entraîner des naissances prématurées et une insuffisance pondérale à la naissance ${ }^{1}$. Chez les mères non anémiques, une supplémentation en fer peut constituer un facteur de protection contre le faible poids à la naissance ${ }^{2}$. Le fer joue également un rôle dans la myélinisation, la fonction des neurotransmetteurs, divers processus cellulaires et oxydatifs, la production d'énergie et le métabolisme des hormones thyroïdiennes ${ }^{1}$. La carence en fer a été mise en cause dans certains troubles neurologiques et cognitifs chez la mère, comme le trouble dépressif majeur, dont les répercussions sur la santé de la mère et de l'enfant sont reconnues ${ }^{3,4}$. Dans ses lignes directrices de 2009, Santé Canada recommande la prise quotidienne d'un supplément de 16 à 20 mg de fer durant la grossesse pour combler les besoins en fer ${ }^{5}$.

Le zinc est essentiel à la synthèse de l'ADN, en plus d'être nécessaire à la structure et à la fonction des protéines régulatrices, structurales et enzymatiques, de même qu'à celles des membranes cellulaires. Il joue un rôle dans la fonction neurologique et favorise la bonne fonction immunitaire ${ }^{1,6,7}$. Diverses études ont associé les naissances prématurées et un faible poids à la naissance à une carence en zinc, bien que la prise systématique de suppléments ne soit pas recommandée, à moins qu'une carence n'ait été constatée ${ }^{8}$. Une carence en zinc a également été mise en cause dans les troubles dépressifs ${ }^{1,4}$. Le folate participe au métabolisme des acides nucléiques et des acides aminés, ainsi qu'au fonctionnement neurologique. Si le folate en quantité insuffisante est responsable de diverses anomalies congénitales et d'issues de

Rattachement des auteurs :

1. Department of Epidemiology and Biostatistics, University of Western Ontario, London (Ontario), Canada

2. Department of Community Health Sciences, University of Calgary, Calgary (Alberta), Canada

3. Department of Family Relations and Applied Nutrition, University of Guelph, Guelph (Ontario), Canada

4. Department of Paediatrics, University of Western Ontario, London (Ontario), Canada

5. Department of Obstetrics and Gynecology, University of Western Ontario, London (Ontario), Canada

6. Children's Health Research Institute, London (Ontario), Canada

7. Lawson Health Research Institute, London (Ontario), Canada

Correspondance : $\mathrm{D}^{\mathrm{re}} \mathrm{M}$. Karen Campbell, Département d'épidémiologie et de biostatistique, immeuble Kresge, pièce K201A, Université Western Ontario, London (Ontario) N6A 5C1; tél. : 519-661-2162; téléc. : 519-661-3766; courriel : Karen.Campbell@schulich.uwo.ca 
grossesse défavorables, c'est toutefois son rôle dans les anomalies du tube neural qui a reçu le plus d'attention. Dans bien des pays, dont le Canada, on conseille aux femmes en âge de procréer de prendre des suppléments. Des politiques d'enrichissement des aliments ont été mises en vigueur, à la lumière de données probantes solides sur l'importance de l'apport en acide folique dès les premiers stades de la gossesse $^{9,10}$. À l'instar du fer et du zinc, la carence en folate est mise en cause dans les troubles dépressifs ${ }^{1,4}$.

Santé Canada a établi un apport nutritionnel recommandé (ANR) pour un certain nombre de nutriments. L'ANR est défini comme " l'apport quotidien moyen permettant de combler les besoins nutritionnels de la quasi totalité (97 à $98 \%$ ) des sujets en bonne santé appartenant à un groupe donné, défini en fonction de l'étape de la vie et du sexe $»^{11}$. Dans le cas des femmes enceintes, l'ANR est de $27 \mathrm{mg} /$ jour pour le fer, de $11 \mathrm{mg} /$ jour pour le zinc et de $600 \mu \mathrm{g} / \mathrm{jour}$, en équivalents de folate alimentaire (EFA), pour le folate ${ }^{11}$. Outre le respect de l'ANR, la Société des obstétriciens et gynécologues du Canada recommande une dose quotidienne de supplément d'acide folique d'au moins $400 \mu g$ (avec des quantités plus élevées indiquées selon le risque) ${ }^{9}$.

Dans cet article, nous examinons l'apport alimentaire quotidien déclaré en fer, en zinc et en folate, estimé à partir des aliments et des suppléments alimentaires consommés par 2019 femmes enceintes de London qui ont participé au Prenatal Health Project (PHP). De plus, nous établissons et examinons les taux d'utilisation de suppléments pour les 2019 femmes et étudions les types de suppléments consommés pour l'ensemble de la cohorte du PHP ( $n=2$ 357). Nous présentons les résultats des analyses qui explorent les déterminants sociodémographiques de l'apport alimentaire.

\section{Méthodologie}

Les données du Prenatal Health Project (PHP) ont été recueillies entre 2002 et 2005 auprès de femmes enceintes recrutées dans des cliniques d'échographie de London (Ontario). Le PHP visait à évaluer les déterminants psychosociaux, nutritionnels, endocriniens et infectieux de la naissance prématurée; sa méthodologie a été expliquée en détail ailleurs ${ }^{12}$. Les critères d'inclusion étaient les suivants : être âgée de 16 ans et plus, vivre dans la grande région de London, présenter une grossesse unique à un stade de 10 à 22 semaines de gestation et parler anglais. Les femmes répondant aux critères d'inclusion et ayant signé le formulaire de consentement étaient admissibles à l'étude. Les femmes enceintes dont le fœtus présentait une anomalie connue étaient exclues.

L'étude a reçu l'aval du comité d'examen éthique pour la recherche en sciences de la santé faisant appel à des sujets humains, de l’Université Western Ontario.

Des intervieweurs qualifiés ont recueilli des données sur l'apport en suppléments alimentaires au moyen du questionnaire d'enquête structuré du PHP administré par téléphone. Ils ont demandé aux répondantes leur nom ainsi que la quantité et la fréquence de consommation de tous les suppléments nutritifs pris régulièrement. Les quantités de nutriments pouvaient être estimées de façon quantitative si la participante mentionnait la marque et le nom d'un supplément prénatal ou, sinon, les quantités exactes de nutriments. Dans les cas où il manquait des renseignements sur la marque ou le produit ( $\mathrm{n}=930$ ), nous avons calculé la composition en nutriments à partir du supplément de multivitamines prénatales le plus utilisé, Centrum Materna. (Sur les 643 femmes de la cohorte principale du PHP qui ont nommé une marque, la plupart avaient utilisé Centrum Materna [ $\mathrm{n}=592$ ], la marque Life [ $\mathrm{n}=24$ ] venant ensuite, et sa composition est identique à celle de Centrum Materna.) Pour calculer des valeurs quotidiennes moyennes, nous avons supposé que la dose standard était d'un comprimé par jour; en cas d'indication contraire, nous avons ajusté les valeurs d'apport en fonction de la fréquence de consommation signalée.

Si l'on peut estimer correctement la teneur en nutriments des produits de multivitamines prénatales, du fait de leur composition similaire, il en est autrement des multivitamines ordinaires pour adultes offertes sur le marché, car leur composition varie énormément. Ainsi, pour les femmes ayant déclaré prendre un supplément de ce type sans en préciser la marque ( $\mathrm{n}=137)$, l'apport nutritionnel fourni par ces suppléments a été déclaré manquant. Pour la même raison, nous n'avons pas non plus tenu compte des suppléments alimentaires renfermant un seul nutriment dont la quantité n'avait pas été spécifiée, à l'exception de l'acide folique. Comme il y a moins de variation entre les suppléments d'acide folique, nous avons supposé que la dose s'établissait à $400 \mu \mathrm{g}$ d'acide folique par comprimé par jour pour les femmes qui n'avaient pas précisé leur dose régulière de ce supplément. Cette hypothèse est conforme à d'autres études ayant permis de mesurer l'apport supplémentaire en folate ${ }^{13}$. En nous basant sur la fréquence de consommation déclarée, nous avons calculé les valeurs d'apport quotidien moyen. Quelques répondantes ont mentionné qu'elles prenaient un supplément d'acide folique qui fournissait plus de $1 \mathrm{mg}$ ( $1000 \mu \mathrm{g}$ ) d'acide folique par jour, mais étant donné la possibilité que cette déclaration soit inexacte, ces renseignements n'ont pas été quantifiés : ils ont été déclarés manquants. L’apport en folate provenant de suppléments a été converti en équivalents de folate alimentaire (EFA) en appliquant un facteur multiplicatif de $1,7^{11}$.

L'apport provenant de l'alimentation a été évalué au moyen d'un questionnaire sur la fréquence de consommation des aliments (QFCA). Ce questionnaire, remis aux participantes à l'étude, devait être rempli avant l'entrevue téléphonique prévue. Les participantes présentaient ensuite leurs réponses durant l'entrevue téléphonique. Le QFCA est considéré comme une méthode acceptable d'évaluation de l'apport alimentaire dans les grandes enquêtes, notamment les études prénatales ${ }^{14}$. Parmi les principaux avantages du QFCA, qui en font un outil plus pratique que les rappels alimentaires ou la tenue de plusieurs journaux alimentaires, mentionnons la réduction du fardeau imposé au répondant et le faible coût de la collecte des données, puisqu'il est possible de l'intégrer facilement à l'entrevue téléphonique même. Comme nous ne disposions d'aucun renseignement précis sur l'apport alimentaire des Canadiennes enceintes, le modèle du QFCA a été fondé sur des 
données recueillies au moyen de rappels alimentaires de 24 heures administrés à 183 femmes qui allaitaient trois mois après l'accouchement ${ }^{15}$. Nous avons comparé le QFCA à un questionnaire créé pour une étude américaine sur la santé prénatale ${ }^{14}$ et nous y avons par la suite ajouté d'autres aliments (p. ex. le bouillon). Un essai pilote du QFCA pour le PHP à été conduit à London en utilisant des journaux alimentaires tenus sur 3 jours par 22 femmes enceintes. Nous avons obtenu les coefficients de corrélation suivants : folate, 0,76 ( $p<0,001)$, zinc, $0,46(p<0,05)$ et fer 0,19 (non significatif).

Le QFCA a permis d'évaluer la fréquence de consommation habituelle de 106 aliments au cours du mois précédant l'entrevue. La fréquence de consommation de chaque aliment a été répartie comme suit : jamais, une fois par jour, 2 ou 3 fois par jour, 4 fois ou plus par jour, une fois par semaine, 2 à 4 fois par semaine, 5 ou 6 fois par semaine, ou 1 à 3 fois par mois. Le logiciel CANDAT Nutrient Calculation System ${ }^{16}$ a été employé pour convertir les réponses en estimations métriques de l'apport énergétique et nutritionnel quotidien, d'après le Fichier canadien sur les éléments nutritifs de $2006^{17}$. Les valeurs d'apport nutritionnel provenant des suppléments ont été ajoutées à celles provenant de l'alimentation pour obtenir l'apport alimentaire quotidien total.

Sur les 3656 femmes rencontrées dans les cliniques d'échographie et invitées à participer au PHP, 2747 ont accepté et 2421 (66\%) ont répondu à l'enquête. De ces 2421 répondantes, 38 ont été exclues de la cohorte longitudinale "de base » à cause de données périnatales non disponibles ou non applicables (pour des raisons telles qu'un suivi impossible, une fausse couche, un avortement ou un décès néonatal). De plus, 26 femmes avaient été recrutées deux fois dans l'étude, pour deux grossesses différentes : l'un des deux ensembles de données a été exclu de façon aléatoire pour chacune de ces participantes. La cohorte longitudinale de base était donc composée de 2357 participantes du PHP, dont 2019 ont été incluses dans les analyses sur les apports présentées dans la présente étude : celles ayant rempli le
QFCA, ayant déclaré un apport énergétique situé au plus à deux écarts-types de la moyenne de l'échantillon (les valeurs situées à l'extérieur de cet intervalle plausible est une indication possible d'une déclaration inexacte), ayant obtenu des valeurs d'apport plausibles tirées du QFCA pour les nutriments d'intérêt et enfin dont les valeurs d'apport en supplément nutritionnel concernant les nutriments d'intérêt ne comportaient aucune donnée manquante.

\section{Analyses statistiques}

Nous avons effectué des analyses descriptives des valeurs estimatives de l'apport quotidien moyen en fer, en zinc et en folate provenant des aliments, des suppléments et de l'apport alimentaire total pour l'ensemble de l'échantillon des 2019 participantes admissibles à l'étude. Pour étudier la contribution de l'utilisation de suppléments, nous avons également calculé les apports moyens par strate pour chaque micronutriment, selon si un supplément contenant le micronutriment était consommé ou non; des tests $t$ de Student ont été effectués pour déterminer si les différences entre les strates d'utilisation de suppléments étaient statistiquement significatives. Nous avons aussi réalisé une matrice de corrélation entre les apports totaux des trois micronutriments pour voir si ces apports étaient liés. Pour évaluer les prédicteurs possibles de l'alimentation, nous avons exploré les associations entre l'alimentation et quatre variables sociodémographiques nominales : l'âge, l'état matrimonial, la scolarité et le revenu du ménage. À cette fin, nous avons effectué des tests d'analyse de la variance pour examiner les associations entre les quatre variables sociodémographiques nominales et chacun des apport alimentaires totaux en fer, en zinc et en folate. De plus, nous avons effectué des tests du chi carré $\left(\chi^{2}\right)$ entre chacune des variables sociodémographiques et dichotomisé l'utilisation de suppléments pour évaluer toute association possible. Enfin, nous avons déterminé les fréquences des types de suppléments alimentaires consommés par toute la cohorte de base du PHP ( $n=2$ 357). Nous avons utilisé le progiciel de statistiques SAS version $9.1^{18}$ pour la gestion des données et les analyses statistiques.

\section{Résultats}

Le tableau 1 présente les caractéristiques des participantes admissibles à l'enquête ( $n=2$ 019). La plupart des femmes étaient âgées de 22 à 34 ans (moyenne d'âge de 30 ans), étaient mariées, avaient obtenu un diplôme collégial ou universitaire et le revenu de leur ménage variait entre 30000 \$ et 79999 \$. La majorité d'entre elles avaient déclaré prendre un ou plusieurs suppléments alimentaires, mais 29,6 \% ne recevaient aucun supplément de zinc, 28,4\% aucun supplément de fer et 20,3 \% aucun supplément d'acide folique. Environ un cinquième de l'échantillon ne consommait aucun produit de multivitamines ordinaires ou prénatales ni de supplément à un seul nutriment contenant du zinc, du fer ou de l'acide folique.

Le tableau 2 présente les analyses descriptives de chacun des trois micronutriments. On y trouve des estimations de l'apport quotidien moyen provenant des aliments, des suppléments alimentaires et des deux sources combinées. On y indique aussi le pourcentage de femmes dont l'apport nutritionnel est inférieur à l'ANR, d'après les estimations de l'apport alimentaire total. En raison des limites inhérentes à la méthode d'évaluation alimentaire du QFCA, il n'est pas jugé approprié de se servir des estimations de l'apport nutritionnel pour évaluer les apports suffisants en nutriments. Toutefois, il est possible d'utiliser les estimations du QFCA pour classer les apports nutritionnels dans une population en fonction de l'ANR ${ }^{19}$. Dans ce cas, un pourcentage relativement élevé de l'échantillon se situait en-dessous de l'ANR pour les trois micronutriments : le fer (31 \%), le zinc (18\%) et le folate (16\%). Une matrice de corrélation entre les apports totaux des trois micronutriments a fait ressortir une forte corrélation.

Le tableau 3 montre les estimations moyennes par strate de l'apport alimentaire total selon la consommation d'un supplément contenant le micronutriment en question. Les tests $t$ de Student correspondants indiquent des différences significatives sur le plan statistique en ce qui concerne les apports moyens pour les trois micronutriments. La figure 1 présente des histogrammes illustrant les distributions 
TABLEAU 1

Analyse de l'apport alimentaire grâce au Prenatal Health Project (PHP) : caractéristiques des participantes $(n=2019)$

\begin{tabular}{|c|c|c|}
\hline \multirow[t]{2}{*}{ Variables nominales } & \multicolumn{2}{|c|}{ Participantes } \\
\hline & $\begin{array}{c}\text { Nombre } \\
\mathbf{n}\end{array}$ & $\begin{array}{c}\text { Pourcentage } \\
\%\end{array}$ \\
\hline \multicolumn{3}{|l|}{ Groupe d'âge (ans) } \\
\hline$<22$ & 85 & 4,2 \\
\hline $22-34$ & 1578 & 78,2 \\
\hline $35+$ & 355 & 17,6 \\
\hline \multicolumn{3}{|l|}{ État matrimonial } \\
\hline Mariée & 1544 & 76,5 \\
\hline Conjointe de fait & 310 & 15,4 \\
\hline Célibataire/séparée/divorcée & 163 & 8,1 \\
\hline \multicolumn{3}{|l|}{ Scolaritéa } \\
\hline Diplôme d'études collégiales/universitaires & 1431 & 71,3 \\
\hline Autre & 575 & 28,7 \\
\hline \multicolumn{3}{|l|}{ Revenu du ménage $\mathrm{a}^{\mathrm{a}}(\$)$} \\
\hline$<30,000$ & 224 & 11,9 \\
\hline $30000-79999$ & 941 & 50,0 \\
\hline $80000+$ & 716 & 38,1 \\
\hline \multicolumn{3}{|c|}{ Prise d'un ou de plusieurs suppléments alimentaires ${ }^{b}$} \\
\hline Oui & 1613 & 79,9 \\
\hline Non & 406 & 20,1 \\
\hline \multicolumn{3}{|l|}{ Prise d'un supplément contenant du fer } \\
\hline Oui & 1446 & 71,6 \\
\hline Non & 573 & 28,4 \\
\hline \multicolumn{3}{|l|}{ Prise d'un supplément contenant du zinc } \\
\hline Oui & 1422 & 70,4 \\
\hline Non & 597 & 29,6 \\
\hline \multicolumn{3}{|c|}{ Prise d'un supplément contenant de l'acide folique } \\
\hline Oui & 1610 & 79,7 \\
\hline Non & 409 & 20,3 \\
\hline Variables mesurées & Moyenne & (Écart-type) \\
\hline Âge (ans) & 30,4 & $(5,0)$ \\
\hline Consommation d'énergie (kcal/jour) & 1982 & (545) \\
\hline
\end{tabular}

a La taille de l'échantillon est ici inférieure à 2019 du fait de données manquantes.

b Contenant de l'acide folique, du fer ou du zinc; celles dont la réponse est « non » pour cette variable ne prenaient donc aucun produit de multivitamines, ni aucun produit renfermant un seul nutriment figurant dans le tableau 4. de ces trois micronutriments. Alors que l'apport total en zinc suit une distribution raisonnablement normale, les distributions pour l'apport total en fer et l'apport total en folate (EFA) sont bimodales, chacune présentant deux pics distincts. Dans le cas de ces deux micronutriments, un pic se trouvait en-dessous du niveau de l'ANR, tandis que l'autre se situait au-dessus. Les pics correspondent aux estimations moyennes par strate du tableau 3; autrement dit, les distributions bimodales sont fonction de l'utilisation de suppléments alimentaires.

Le tableau 4 détaille les types de suppléments alimentaires utilisés, c'està-dire les multivitamines et les suppléments à un seul nutriment contenant du fer, du zinc ou du folate. Pour montrer la gamme complète des produits utilisés, les chiffres reposent sur l'ensemble de la cohorte de base du PHP composée de 2357 femmes. Par conséquent, le tableau comprend des entrées qui n'ont pu être quantifiées. Parmi les suppléments de multivitamines prénatales spécifiés, le produit le plus couramment utilisé était Centrum Materna ( $\mathrm{n}=592$ ). Dans le cas de suppléments contenant des micronutriments précis (suppléments renfermant un seul nutriment ou produits contenant un petit nombre de nutriments), les plus courants étaient les suppléments d'acide folique ( $\mathrm{n}=354$ ), suivis des suppléments de fer $(\mathrm{n}=98)$.

Des tests d'analyse de la variance ont été réalisés dans le but de déterminer s'il existait des associations entre les quatre variables sociodémographiques nominales et chacun des apports alimentaires totaux en fer, en zinc et en folate. Aucun résultat n'était statistiquement significatif. De même, des tests du $\chi^{2}$ ont été effectués

TABLEAU 2

Apport en fer, en zinc et en folate provenant des aliments et des suppléments alimentaires des femmes enceintes $(n=2019)$

\begin{tabular}{|c|c|c|c|c|c|}
\hline \multirow[t]{2}{*}{ Micronutriment } & \multirow{2}{*}{$\begin{array}{c}\text { ANR pour les femmes } \\
\text { enceintes }\end{array}$} & \multicolumn{3}{|c|}{ Apport quotidien moyen estimatif, poids par jour (ÉT) } & \multirow{2}{*}{$\begin{array}{c}\text { Proportion de } \\
\text { l'échantillon en-dessous } \\
\text { de l'ANR, \% }\end{array}$} \\
\hline & & Aliments seulement & $\begin{array}{l}\text { Suppléments } \\
\text { alimentaires seulement }\end{array}$ & Total & \\
\hline Fer (mg/jour) & 27 & (4) & $(12)$ & $32 \quad(13)$ & 31 \\
\hline Zinc (mg/jour) & 11 & (3) & (3) & 16 & 18 \\
\hline Folate $(\mu \mathrm{g} /$ jour EFA) & 600 & 473 (155) & $1338^{a} \quad(763)$ & 1811 (772) & 16 \\
\hline
\end{tabular}

Abréviations : EFA, équivalent folate alimentaire; ANR, apport nutritionnel recommandé; ÉT, écart-type.

${ }^{a}$ Outre l'ANR par les aliments, la Société des obstétriciens et gynécologues du Canada recommande aux femmes enceintes de prendre un supplément d'acide folique de $400 \mu$ ğ $(400 \mu \mathrm{g} \text { d'acide folique }=\text { environ } 680 \mathrm{EFA})^{9}$. 
TABLEAU 3

Apport alimentaire total des femmes enceintes en fer, en zinc et en folate, stratifié en fonction de l'utilisation de suppléments ( $\mathbf{n}=\mathbf{2} 019$ )

\begin{tabular}{|c|c|c|c|c|}
\hline \multirow[t]{2}{*}{ Micronutriment } & \multirow[t]{2}{*}{$\begin{array}{l}\text { ANR pour les femmes } \\
\text { enceintes }\end{array}$} & \multicolumn{2}{|c|}{$\begin{array}{l}\text { Apport quotidien moyen estimatif, poids par jour (ÉT) } \\
\text { [n] }\end{array}$} & \multirow[t]{2}{*}{ Test $t$ de Student } \\
\hline & & $\begin{array}{l}\text { Participantes dont les } \\
\text { micronutriments } \\
\text { proviennent uniquement } \\
\text { des aliments }\end{array}$ & $\begin{array}{l}\text { Participantes dont les } \\
\text { micronutriments } \\
\text { proviennent des aliments } \\
\text { et des suppléments }\end{array}$ & \\
\hline Fer (mg/jour) & 27 & $13 \quad(4)$ & $40 \quad(6)$ & $-100,0$ \\
\hline Zinc (mg/jour) & & [n = 597] & {$[n=1422]$} & \\
\hline \multirow[t]{2}{*}{ Folate ( $\mu \mathrm{g} /$ jour EFA) } & 600 & $482(157)$ & $2148(422)$ & $-78,4$ \\
\hline & & {$[n=409]$} & {$[n=1610]$} & \\
\hline
\end{tabular}

Abréviations : EFA, équivalent folate alimentaire; ÉT, écart-type; $p$, valeur $\mathrm{p}$.

${ }^{*} p<0,001$

FIGURE 1

Estimations de l'apport alimentaire en folate, en fer et en zinc provenant des aliments et des suppléments (séparément et combinés) chez les participantes ( $\mathrm{n}=2019$ femmes enceintes) à l'enquête du Prenatal Health Project (PHP)
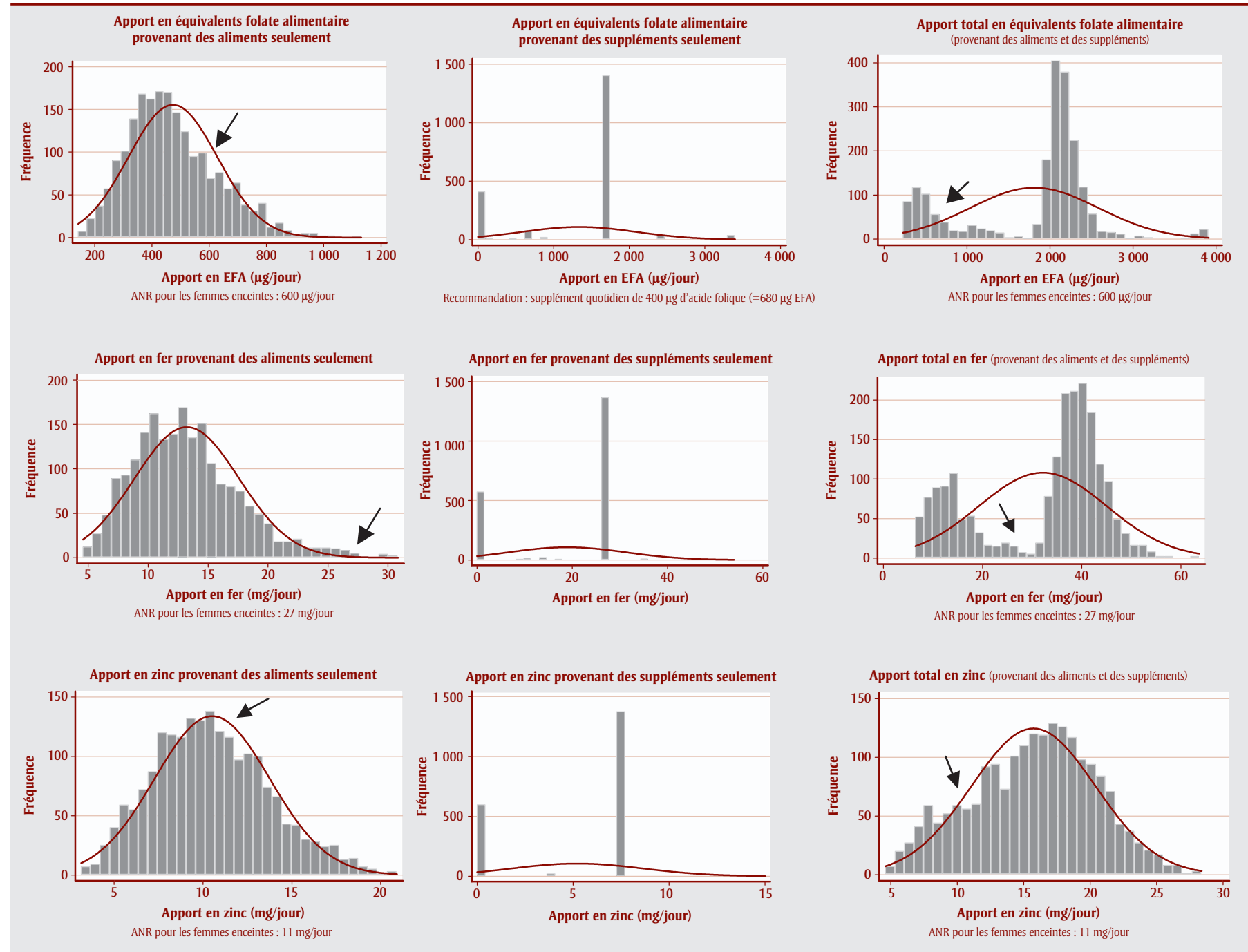

Abréviations : ANR, apport nutritionnel recommandé; EFA, équivalent folate alimentaire.

Remarque : Les flèches indiquent approximativement l'ANR pour chaque nutriment. 
entre chacune des variables sociodémographiques et l'utilisation de suppléments alimentaire dichotomisée : aucun n’a donné de résultats significatifs sur le plan statistique. Autrement dit, ni l'âge, ni l'état matrimonial, ni la scolarité, ni le revenu du ménage n'étaient associés à l'apport alimentaire total ou à l'utilisation de suppléments dans ce groupe de femmes.

\section{Analyse}

La ville de London est située au sud-ouest de l'Ontario. En 2006, sa population dépassait tout juste les 350000 habitants $^{20}$. En 2005, le revenu familial médian déclaré était de 67018 \$, soit à peine plus élevé que celui pour l'ensemble du Canada (63 866 \$) et légèrement plus bas que celui de l'Ontario (69 $156 \$)^{20}$. Les résultats de cette étude pourraient donc s'avérer instructifs pour les autres villes canadiennes ayant des caractéristiques similaires.

Peu d'études canadiennes ont examiné l'apport alimentaire et l'utilisation de suppléments durant la grossesse. Celles qui l'ont fait visaient surtout l'utilisation de suppléments d'acide folique ${ }^{21-25}$. En ce qui a trait à l'apport nutritionnel provenant

TABLEAU 4

Suppléments de multivitamines et suppléments renfermant un seul nutriment comprenant du fer, du zinc ou du folate que les participantes ( $n=2357$ femmes) de la cohorte du Prenatal Health Project (PHP) ont déclaré avoir consommés

\begin{tabular}{|c|c|c|c|}
\hline \multirow[t]{2}{*}{ Source } & \multicolumn{3}{|c|}{$\begin{array}{c}\text { Nombre d'entrées autodéclarées } \\
\text { n }\end{array}$} \\
\hline & Totales & Quantifiées & Manquantes \\
\hline \multicolumn{4}{|l|}{ Supplément de multivitamines ordinaires } \\
\hline Produit spécifiéa & 37 & 37 & 0 \\
\hline Produit non spécifié & 137 & 0 & $137^{\mathrm{b}}$ \\
\hline \multicolumn{4}{|l|}{ Supplément de multivitamines prénatales } \\
\hline Produit spécifié : Centrum Materna & 592 & 592 & 0 \\
\hline Produit spécifié : autre ${ }^{c}$ & 51 & 51 & 0 \\
\hline Produit non spécifié & 930 & $930^{d}$ & 0 \\
\hline \multicolumn{4}{|l|}{ Fer } \\
\hline Supplément ne contenant que du fer & 95 & 31 & $64^{\mathrm{e}}$ \\
\hline Supplément de fer + un autre micronutriment & 3 & 2 & $1^{e}$ \\
\hline \multicolumn{4}{|l|}{ Zinc } \\
\hline Supplément ne contenant que du zinc & 2 & 2 & 0 \\
\hline Zinc + quelques autres micronutriments choisis & 1 & 0 & $1^{e}$ \\
\hline \multicolumn{4}{|l|}{ Folate } \\
\hline Supplément ne contenant que de l'acide folique & 347 & 315 & 32 \\
\hline $\begin{array}{l}\text { Supplément d'acide folique + quelques autres } \\
\text { micronutriments choisis }\end{array}$ & 7 & 3 & 4 \\
\hline
\end{tabular}

Remarque : Ce tableau montre les fréquences d'autodéclaration des types de suppléments. Pour présenter la gamme complète des produits utilisés, la cohorte de base du PHP a été prise en compte, y compris les participantes qui ont été exclues des autres analyses de cette étude. Il est à noter que certaines femmes peuvent avoir pris de nombreux types de suppléments; il est donc possible qu'il y ait plusieurs entrées pour une même participante. À l'opposé, une proportion remarquable de femmes ne prenait aucun supplément (voir texte et tableau 1), ce qui explique qu'il n'y ait aucune entrée pour ces participantes.

${ }^{a}$ Marques de multivitamines ordinaires spécifiées : Centrum (ordinaire), Centrum Forte, Centrum Protegra, Pierrafeu (multivitamines pour enfants), Nutrilite Double X, Life Daily One for Women, Life Spectrum, Life Spectrum Forte, One A Day, One A Day - Femmes.

${ }^{\mathrm{b}}$ Donnée déclarée manquante car, contrairement aux multivitamines prénatales, la composition en nutriments des multivitamines ordinaires pour adultes varie énormément d'un produit à l'autre. On ne peut donc pas la déduire d'une autre marque.

c Marques de multivitamines prénatales spécifiées (à l'exception de Centrum Materna) : Equate, Fem, GNC, Jamieson, Life, Natural Factors (MultiStart), Orifer F, PregVit, Rexall, Thorne Research, Truly.

${ }^{\mathrm{d}}$ On suppose qu'elle est identique à Materna puisque les compositions en nutriments des différents produits de multivitamines prénatales sont très semblables.

e Donnée déclarée manquante parce que la composition en nutriments des suppléments alimentaires renfermant un seul nutriment (sauf l'acide folique) varie énormément d'un produit à l'autre. On ne peut donc pas la déduire à partir d'autres marques. des aliments chez les Canadiennes enceintes, une étude de Pick et collab. ${ }^{26}$ réalisée en 2005 portait sur les niveaux d'apport nutritionnel d'un petit échantillon de femmes enceintes et de femmes non enceintes d'Edmonton, en Alberta. Comme les auteurs faisaient état d'observations tirées d'une étude pilote, la taille de l'échantillon était relativement faible ( $\mathrm{n}=52$ femmes enceintes), ce qu'ils ont reconnu comme étant une limite ${ }^{26}$. À l'opposé, notre étude utilise un échantillon de très grande taille et un instrument validé, ayant fait l'objet d'un projet pilote, pour saisir les données sur l'apport alimentaire. De plus, Pick et collab. n'ont pas tenu compte des valeurs nutritionnelles provenant des suppléments alimentaires ${ }^{26}$, alors que nous avons été capables d’intégrer les valeurs nutritionnelles des suppléments pour produire les estimations de l'apport total. Par conséquent, notre étude offre un aperçu valable de l'état nutritionnel d'une population de femmes enceintes au Canada, tout en contribuant à la littérature dans ce domaine.

Nous avons constaté chez une proportion importante de femmes des apports alimentaires en fer, en zinc et en folate inférieurs aux valeurs de l'ANR. L'apport nutritionnel provenant uniquement des aliments était particulièrement faible (figure 1). Ces observations étayent d'autres études canadiennes selon lesquelles il est difficile pour les femmes enceintes, à partir uniquement de l'alimentation, de suivre les recommandations concernant les principaux micronutriments ${ }^{26,27}$.

En outre, un cinquième des femmes ne prenait aucun supplément contenant l'un ou l'autre des trois micronutriments. Compte tenu de l'importance de ces éléments pour la santé de la mère et du fœetus, ces résultats sont préoccupants.

Les lignes directrices de pratique clinique insistent sur l'importance de la supplémentation en acide folique durant la grossesse ${ }^{9}$ et recommandent la supplémentation en fer ${ }^{5}$. Il n'est donc pas étonnant que ces deux micronutriments aient été les plus utilisés parmi les produits renfermant un seul micronutriment. Les distributions bimodales associées à ces deux micronutriments sont fonction de l'utilisation de suppléments 
alimentaires, comme l'illustre le tableau 3; les femmes qui avaient utilisé des suppléments de ces micronutriments dépassaient largement les ANR les concernant et représentaient les pics de valeurs élevées, tandis que celles qui n'avaient pas pris de suppléments alimentaires n'avaient pas atteint les ANR les concernant et représentaient les pics de valeurs basses. Par conséquent, l'utilisation de suppléments alimentaires est de toute évidence essentielle pour atteindre les niveaux d'apport en micronutriments nécessaires durant la grossesse.

Malgré l'enrichissement en acide folique des aliments au Canada et aux États-Unis, la plupart des femmes semblent avoir besoin d'un supplément distinct d'acide folique pour obtenir les concentrations de folate dans les globules rouges nécessaires à la prévention des anomalies du tube neural ${ }^{25,27}$. Bien qu'on ignore le mécanisme exact par lequel l'acide folique prévient ce type d'anomalies, il semble que les suppléments d'acide folique (plutôt que les folates naturellement présents dans les aliments) seraient la clé de l'effet préventif ${ }^{25}$. Il est donc inquiétant de constater que $20 \%$ des femmes composant l'échantillon ne prenaient aucun supplément alimentaire contenant de l'acide folique.

Comme nous l'avons mentionné à la section Résultats, les valeurs d'apport pour les trois micronutriments étaient en général très corrélées. Ces résultats s'expliquent peut-être par le fait que les gens présentent souvent des carences en de nombreux micronutriments en raison de mauvaises habitudes alimentaires générales; ils pourraient aussi être attribuables à l'utilisation de suppléments de multivitamines, qui font en sorte que les personnes obtiennent les micronutriments sous une forme combinée.

L'âge, l'état matrimonial, la scolarité et le revenu du ménage n'étaient pas associés à l'apport alimentaire total en micronutriments ni à l'utilisation de suppléments. Dans le cadre d'une analyse distincte faisant appel aux données du PHP, nous avons utilisé une régression multivariée pour évaluer les prédicteurs de l'apport alimentaire en zinc de façon plus poussée. Ces observations, faites dans le contexte d'une question de recherche sur les prédicteurs de la dépression prénatale, ont été exposées en détail ailleurs ${ }^{20,28}$. Aucune des variables sociodémographiques ni le stress psychosocial n'étaient considérés comme des prédicteurs de l'apport nutritionnel en zinc dans cette cohorte ${ }^{28}$. Dans son ensemble, la cohorte bénéficie d'un statut socio-économique plus élevé que la population générale de la ville de London ${ }^{20,28}$. Par conséquent, d'autres facteurs expliquent peut-être la variation observée dans l'apport alimentaire. Il serait pertinent, sous l'angle de la politique de santé publique, de pousser l'examen plus loin pour mettre au jour ces facteurs. Il va sans dire que le lien entre le statut socioéconomique et l'apport alimentaire est bien établi ${ }^{29-33}$. À cet égard, il est un peu étonnant de constater qu'une proportion remarquable de femmes appartenant à une cohorte plus socialement favorisée présente un apport alimentaire insuffisant et qu'elles n'utilisent pas de suppléments. De tels résultats peuvent mettre en évidence l'existence d'obstacles supplémentaires dans la vie des Canadiennes, qui ne sont pas révélés par les indicateurs habituels de statut socio-économique. Les publications sur les suppléments d'acide folique, par exemple, laissent entendre que les obstacles pourraient se situer au niveau des politiques de santé publique et des fournisseurs de soins de santé24,25. On s'intéresse de plus en plus aux déterminants sociaux de la santé de la population et à la promotion de la santé sous l'angle de la santé publique ${ }^{34-36}$, et ces deux dimensions peuvent nous aider à comprendre les déterminants de l'apport alimentaire et de l'utilisation de suppléments chez les Canadiennes en âge de procréer. Il y a toutefois lieu de pousser plus loin les recherches et les interventions afin de promouvoir efficacement de saines habitudes alimentaires dans tous les segments de la population canadienne.

\section{Points forts et limites de l'étude}

La méthode d'évaluation de l'apport alimentaire au moyen du QFCA ne donne qu'une estimation des valeurs nutritionnelles, ce qui empêche de déterminer avec certitude le caractère adéquat de l'apport pour chaque personne. Toutefois, pour les vastes enquêtes comme celle-ci, il s'agit d'une méthode acceptable et utile, qui offre tout même un aperçu pertinent pour la santé publique. Comme il est mentionné à la section Méthodologie, le coefficient de corrélation pour la validation de l'apport en fer est faible $(0,19)$. Comme l'estimation de l'apport en fer tirée des données du QFCA était plus faible que celle obtenue à partir des journaux alimentaires sur 3 jours durant l'étude pilote, nos résultats sont probablement prudents. Toutefois, ce problème ne tient probablement pas compte de la différence très marquée de l'apport total en fer entre les femmes qui prennent un supplément renfermant du fer et celles qui n'en prennent pas. Autrement dit, cela ne change rien à la conclusion selon laquelle l'utilisation de suppléments est importante pour obtenir l'ANR en fer durant la grossesse.

Comme nous l'avons précisé à la section Méthodologie, nous avons supposé que les suppléments alimentaires prénatals non spécifiés avaient une composition similaire à ceux de la marque Centrum Materna. Cette hypothèse est raisonnable, vu la similarité entre les produits de multivitamines prénatales populaires offerts sur le marché. De plus, pour celles qui n'avaient pas précisé la quantité de leur supplément d'acide folique, nous avons supposé une dose quotidienne de 400 rg d'acide folique, même si les suppléments d'acide folique sont offerts à des doses plus élevées. Une démarche semblable a été utilisée dans d'autres études visant à mesurer ce type de produit. La différence dans les estimations moyennes des EFA est assez marquée entre les femmes qui prennent des suppléments d'acide folique et celles qui n'en prennent pas; par conséquent, une sous-estimation possible, attribuable à cette supposition, de l'apport en acide folique pour quelques participantes ne changerait rien à nos conclusions sur l'importance de la supplémentation en acide folique pour obtenir l'ANR en folate.

Les principaux points forts de cette étude sont la grande taille de l'échantillon, le fait que ce dernier est basé sur la collectivité et l'évaluation exhaustive des sources (aussi bien aliments que suppléments) pour l'estimation de l'apport alimentaire. Parmi les points faibles, citons l'existence possible d'un biais de sélection et d'un biais dans 
les réponses. Ces biais sont inévitables, même si nous avons fait ce qu'il fallait pour les réduire. Nous avons réduit le biais dans les réponses grâce à des intervieweurs téléphoniques qualifiés qui devaient guider les participantes à travers toutes les composantes du questionnaire, y compris le QFCA. Quant au biais de sélection, nous l'avons réduit en recrutant dans des cliniques d'échographie situées un peu partout à London, les femmes enceintes s'y rendant systématiquement pour passer une échographie. Une des limites possibles est le fait que seules des femmes anglophones pouvaient être recrutées. Cependant, comme moins de $2 \%$ des femmes de London ne parlent pas anglais ${ }^{20}$, cette restriction a un effet probablement négligeable. La cohorte du PHP bénéficie d'un statut socio-économique un peu plus élevé que la population générale de la ville de London ${ }^{20}$, ce qui peut révéler un biais de sélection; toutefois, cette situation a également permis de mettre en lumière des observations étonnantes au sujet de l'apport alimentaire et de l'utilisation de suppléments dans des segments de la population socialement plus favorisés, comme nous l'avons mentionné plus haut.

\section{Conclusions}

Même si l'importance générale de l'utilisation de suppléments de multivitamines prénatales est reconnue dans les lignes directrices de pratique clinique9, le zinc n'est pas considéré comme un micronutriment d'intérêt. Si la supplémentation en acide folique et en fer est formellement recommandée, les données de notre étude semblent indiquer que, dans la population, la consommation de nutriments en quantité suffisante et l'utilisation de suppléments sont loin d'être acquises. Dans cette cohorte, une proportion remarquable de femmes présentaient des apports alimentaires insuffisants pour les trois micronutriments et ne prenaient aucun supplément, même si elles bénéficiaient d'un statut socio-économique globalement plus élevé. Il y a tout lieu de poursuivre les recherches en vue d'évaluer à la fois le degré de généralisation possible et le succès de la mise en œuvre dans la population des recommandations actuelles de supplémentation durant la grossesse.

\section{Remerciements}

Les auteurs souhaitent remercier M. Larry Stitt pour ses conseils concernant le logiciel SAS version 9.1. Le Prenatal Health Project a reçu un appui financier des Instituts de recherche en santé du Canada (IRSC). Les auteurs déclarent n'avoir aucun conflit d'intérêt.

\section{Références}

1. Allen LH. Biological mechanisms that might underlie iron's effects on fetal growth and preterm birth. J Nutr. 2001 Feb;131(2S-2):581S-9S.

2. Palma S, Perez-Iglesias R, Prieto D, Pardo R, Llorca J, Delgado-Rodriguez M. Iron but not folic acid supplementation reduces the risk of low birthweight in pregnant women without anaemia: a case-control study. J Epidemiol Community Health. 2008 Feb;62 (2):120-4.

3. Bodnar LM, Wisner KL. Nutrition and depression: implications for improving mental health among childbearing-aged women. Biol Psychiatry. 2005 Nov 1;58(9):679-85.

4. Leung BM, Kaplan BJ. Perinatal depression: prevalence, risks, and the nutrition link-a review of the literature. J Am Diet Assoc. 2009 Sep;109(9):1566-75.

5. Groupe consultatif d'experts sur les Lignes directrices nationales sur la nutrition pendant la grossesse. Lignes directrices sur la nutrition pendant la grossesse à l'intention des professionnels de la santé Le fer contribue à une grossesse en santé. Ottawa (Ont.) : Santé Canada; 2009 (H164-109/1-2009F-PDF au catalogue).

6. Fraker PJ, King LE, Laakko T, Vollmer TL. The dynamic link between the integrity of the immune system and zinc status. J Nutr. 2000 May;130(5S Suppl):1399S-406S.

7. Huang EP. Metal ions and synaptic transmission: think zinc. Proc Natl Acad Sci USA. 1997 Dec 9;94(25):13386-7.

8. Ladipo OA. Nutrition in pregnancy: mineral and vitamin supplements. Am J Clin Nutr. 2000 Jul;72 (1 Suppl):280S-90S.
9. Wilson RD. Supplémentation préconceptionnelle en vitamines/acide folique 2007 : Utilisation d'acide folique, conjointement avec un supplément multivitaminique, pour la prévention des anomalies du tube neural et d'autres anomalies congénitales. Directive clinique no 201. Ottawa (Ont.) : Société des obstétriciens et gynécologues du Canada, déc. 2007; une publication conjointe du programme Motherisk, Hospital for Sick Children, Toronto (Ont.).

10. Wilson RD, Davies G, Desilets V, Reid GJ, Summers A, Wyatt P et collab. The use of folic acid for the prevention of neural tube defects and other congenital anomalies. J. obstet. gynécol. J Obstet Gynaecol Can. 2003 Nov;25(11):959-73.

11. Santé Canada. Tableaux des apports nutritionnels de référence [Internet]. Ottawa (Ont.) : Santé Canada; 2006 [consultation le $1^{\text {er }}$ septembre 2007]. Consultable à partir de la page : http:// www.hc-sc.gc.ca/fn-an/nutrition/reference /table/index-fra.php

12. Sontrop JM, Campbell MK, Evers SE, Speechley KN, Avison WR. Fish consumption among pregnant women in London, Ontario: associations with socio-demographic and health and lifestyle factors. Can J Public Health. 2007 Sep;98(5):389-94.

13. Stevens VL, Rodriguez C, Pavluck AL, McCullough ML, Thun MJ, Calle EE. Folate nutrition and prostate cancer incidence in a large cohort of US men. Am J Epidemiol. 2006 Jun 1;163(11):989-96.

14. Brown JE, Buzzard IM, Jacobs DR Jr., Hannan PJ, Kushi LH, Barosso GM et collab. A food frequency questionnaire can detect pregnancy-related changes in diet. J Am Diet Assoc. 1996 Mar;96(3):262-6.

15. Doran L, Evers S. Energy and nutrient inadequacies in the diets of low-income women who breast-feed. J Am Diet Assoc. 1997 Nov;97(11):1283-7.

16. CANDAT. London (Ont.): Godin London Inc.; 2004. Consultation à partir de la page : http://www.candat.ca 
17. Fichier canadien sur les éléments nutritifs 2006 [Internet]. Ottawa (Ont.) : Santé Canada; 2006 [consultation le 20 novembre 2010]. Consultable à partir de la page : http:// www.hc-sc.gc.ca/fn-an/nutrition /fiche-nutri-data/cnf_aboutus-aproposdenous _fcen-fra.php

18. SAS Institute Inc. Base SAS 9.1 Procedures Guide. Carey (NC): SAS Institute Inc.; 2004.

19. Institute of Medicine. Dietary reference intakes: applications in dietary assessment. Washington (DC): National Academy Press; 2000.

20. Statistique Canada. Profils des communautés de 2006 : London, Ontario [Internet]. Ottawa (Ont.) : Statistique Canada; 2006 [consultation le $1^{\mathrm{er}}$ février 2011]. Consultable en ligne à partir de la page : http:// www12.statcan.ca/census-recensement /2006/dp-pd/prof/92-591/index.cfm?Lang = F

21. Han A, Rotermann M, Fuller-Thomson E, Ray JG. Pre-conceptional folic acid supplement use according to maternal country of birth. J Obstet Gynaecol Can. 2009 Mar;31(3):222-6.

22. Morin P, De WP, Noiseux M, Niyonsenga T, St-Cyr-Tribble D, Tremblay C. Pregnancy planning and folic acid supplement use: results from a survey in Quebec. Prev Med 2002 Aug;35(2):143-9.

23. Morin P, De Wals P, St-Cyr-Tribble D, Niyonsenga T, Payette $H$. Pregnancy planning: a determinant of folic acid supplements use for the primary prevention of neural tube defects. Can J Public Health. 2002 Jul;93(4):259-63.

24. Morin P, Demers K, Giguere C, Tribble DS, Lane J. Prise de suppléments multivitaminiques pour la prévention primaire des anomalies congénitales : application d'une pratique clinique préventive. Med. fam. can. Déc. 2007; 53(12):2142-3.

25. Reisch HS, Flynn MA. Folic acid and the prevention of neural tube defects (NTDs). Challenges and recommendations for public health. Rev. can. santé publique = Can J Public Health. 2002 Jul;93(4):254-8.

26. Pick ME, Edwards M, Moreau D, Ryan EA. Assessment of diet quality in pregnant women using the Healthy Eating Index. J Am Diet Assoc. 2005 Feb;105(2):240-6.
27. Shuaibi AM, House JD, Sevenhuysen GP. Folate status of young Canadian women after folic acid fortification of grain products. J Am Diet Assoc. 2008 Dec;108(12):2090-4.

28. Roy A, Evers SE, Avison WR, Campbell MK. Higher zinc intake buffers the impact of stress on depressive symptoms in pregnancy. Nutr Res. 2010 Oct;30(10):695-704.

29. Billson H, Pryer JA, Nichols R. Variation in fruit and vegetable consumption among adults in Britain. An analysis from the dietary and nutritional survey of British adults. Eur J Clin Nutr. 1999 Dec;53(12):946-52.

30. Hall KD, Stephen AM, Reeder BA, Muhajarine N, Lasiuk G. Diet, obesity and education in three age groups of Saskatchewan women. Can J Diet Pract Res. 2003;64(4):181-8.

31. Irala-Estevez JD, Groth $\mathrm{M}$, Johansson $\mathrm{L}$, Oltersdorf U, Prattala R, MartinezGonzalez MA. A systematic review of socio-economic differences in food habits in Europe: consumption of fruit and vegetables. Eur J Clin Nutr. 2000 Sep;54(9):706-14.

32. Janssen I, Boyce WF, Simpson K, Pickett W. Influence of individual- and area-level measures of socioeconomic status on obesity, unhealthy eating, and physical inactivity in Canadian adolescents. Am J Clin Nutr. 2006 Jan;83(1):139-45.

33. Jasti S, Siega-Riz AM, Bentley ME. Dietary supplement use in the context of health disparities: cultural, ethnic and demographic determinants of use. J Nutr. 2003 Jun;133(6):2010S-3S.

34. CDSS. Combler le fossé en une génération : instaurer l'équité en santé en agissant sur les déterminants sociaux de la santé : rapport final de la Commission des Déterminants Sociaux de la Santé. Genève $(\mathrm{CH})$ : Organisation mondiale de la Santé; 2009.

35. Agence de la santé publique du Canada. Pourquoi les Canadiens sont-ils en santé ou pas? Ottawa (Ont.) : Agence de la santé publique du Canada; 2003 [consultation le 20 nov. 2010]. Consultable en ligne à la page : http://www.phac-aspc.gc.ca/ph-sp /determinants/determinants-fra.php
36. Organisation mondiale de la Santé. Charte d'Ottawa pour la promotion de la santé [Internet]. Genève $(\mathrm{CH})$ : Organisation mondiale de la Santé; 1986 [consultation le 10 sept. 2008]. PDF (en anglais, 17 Ko) téléchargeable à partir du lien : http:// ww w. who.int / h pr / N P H / d ocs / ottawa_charter_hp.pdf 